\title{
Slant Curves in 3-dimensional Normal Almost Contact Geometry
}

\section{Constantin Călin \& Mircea Crasmareanu}

Mediterranean Journal of Mathematics

ISSN 1660-5446

Mediterr. J. Math.

DOI 10.1007/s00009-012-0217-1

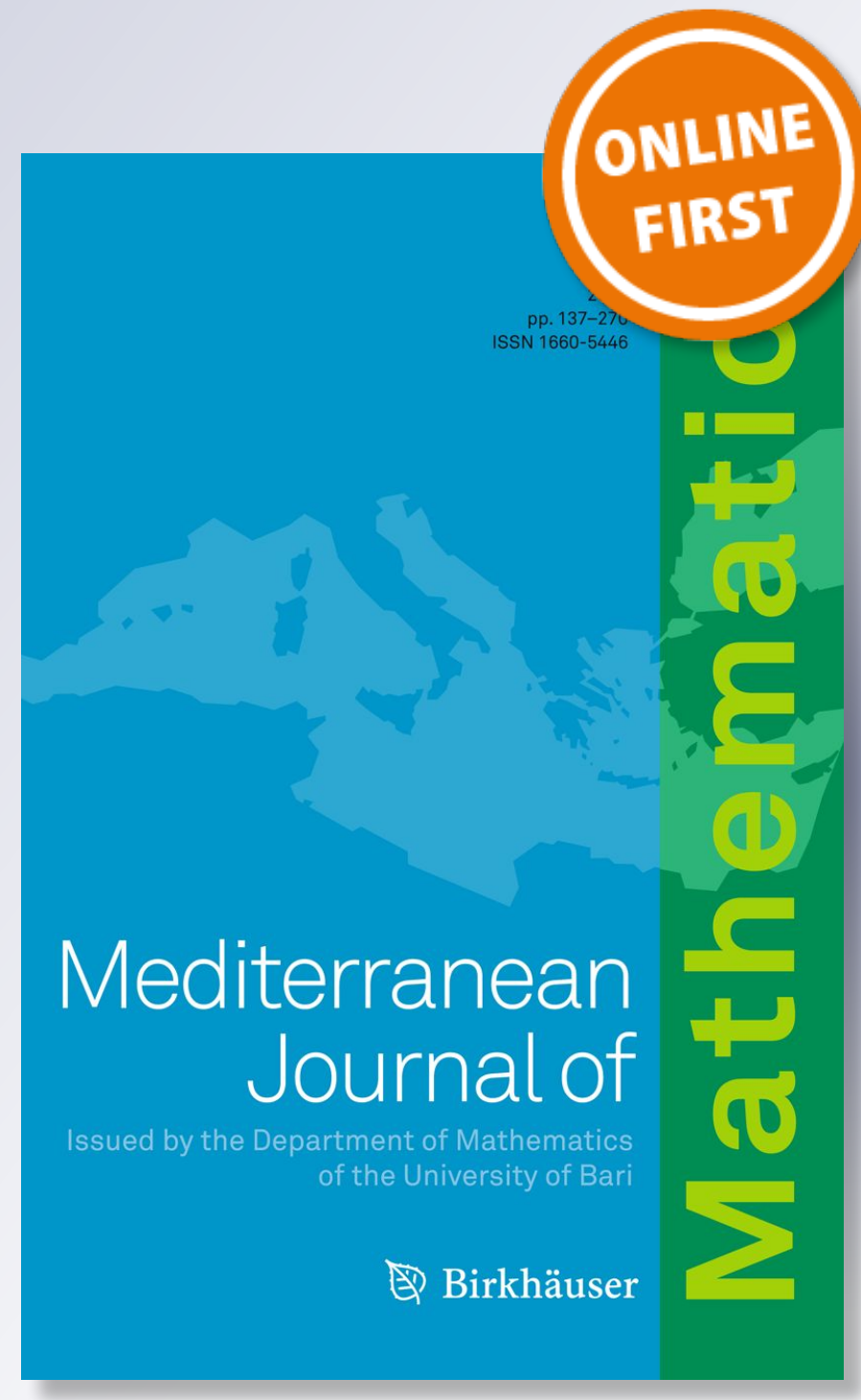

算 Springer 
Your article is protected by copyright and all rights are held exclusively by Springer Basel AG. This e-offprint is for personal use only and shall not be self-archived in electronic repositories. If you wish to self-archive your work, please use the accepted author's version for posting to your own website or your institution's repository. You may further deposit the accepted author's version on a funder's repository at a funder's request, provided it is not made publicly available until 12 months after publication. 


\title{
Slant Curves in 3-dimensional Normal Almost Contact Geometry
}

\author{
Constantin Călin* and Mircea Crasmareanu
}

\begin{abstract}
The aim of this paper is to study slant curves of three-dimensional normal almost contact manifolds as natural generalization of Legendre curves. Such a curve is characterized by means of the scalar product between its normal vector field and the Reeb vector field of the ambient space. In the particular case of a helix we show that it has a proper (non-harmonic) mean curvature vector field. The general expressions of the curvature and torsion of these curves and the associated Lancret invariant are computed as well as the corresponding variants for some particular cases, namely $\beta$-Sasakian and cosymplectic. A class of examples is discussed for a normal not quasi-Sasakian 3-manifold.
\end{abstract}

Mathematics Subject Classification (2010). Primary 53D15; Secondary 53B25, 53A $55,53 \mathrm{C} 25$.

Keywords. Normal almost contact manifold, slant curve, Legendre curve, Lancret invariant.

\section{Introduction}

A nice notion of classical differential geometry of curves is that of curve of constant slope, also called cylindrical helix. This is a curve in the Euclidean space $\mathbb{E}^{3}$ for which the tangent vector field has a constant angle with a fixed direction called the axis. The second name corresponds to the fact that there exists a cylinder on which the curve moves in such a way that it cuts each ruling at a constant angle. The classical characterization of these curves is the Bertrand-Lancret-de Saint Venant Theorem ([2]): the curve $\gamma$ in $\mathbb{E}^{3}$ is of constant slope if and only if the ratio of the torsion $\tau$ and the curvature $k$ is constant. More precisely, for a cylindrical helix we have the constant ratio $\frac{\cos \theta}{|\sin \theta|}=\frac{\tau}{k}$ and then, inspired by the title of [2], we define the Lancret invariant as $\operatorname{Lancret}(\gamma)=\frac{\cos \theta}{|\sin \theta|}$. By computing $k$ and $\tau$ in terms of $\theta$ we

*Corresponding author. 


\section{Călin and M. Crasmareanu}

get the result above and therefore the expression of Lancret invariant in the 3-dimensional Euclidean geometry is:

$$
\operatorname{Lancret}(\gamma)=\frac{\tau}{k}
$$

An interesting generalization of this class of curves is that of slant curve in almost contact metric geometry. This concept was introduced in [9] with the constant angle $\theta$ between the tangent and the Reeb vector field. The particular case of $\theta=\frac{\pi}{2}$ ( or $\theta=\frac{3 \pi}{2}$ ) is very important since we recover the Legendre curves of [1]. For the same general expression $\frac{\cos \theta}{|\sin \theta|}$, Theorem 3.1 of $[9$, p. 362] gives the form of Lancret invariant in 3-dimensional Sasakian geometry:

$$
\operatorname{Lancret}(\gamma)=\frac{\tau \pm 1}{k}
$$

Although the literature on Legendre curves is rich ([3],[5],[6],[11],[14], [15],[17],[18]), slant curves have been studied until now only for the Sasakian geometry in [9], for the contact pseudo-Hermitian geometry in [10] and for the $f$-Kenmotsu geometry in [8]. So, the purpose of this paper is to begin a study of slant curves in another important class of almost contact manifolds namely normal, which are provided by the integrability of an almost complex structure naturally associated to the given almost contact structure.

Our work is structured as follows: the first section is a very brief review of (normal) almost contact geometry and Frenet curves in general Riemannian geometry. The next section is focused on the study of slant (particularly Legendre) curves in this generalized framework. So, we obtain a characterization of slant curves similar to Proposition 3.1 of $[9$, p. 362] and the form of Lancret invariant is derived. Our expression for Lancret invariant is more complex, involving a square root in addition to curvature and torsion, and the derivative of a function along the curve. Another main result of this section is that a helix slant curve has a proper (non-harmonic) curvature vector field. The last section is devoted to examples and two such curves are completely described: the first one is a generalized helix for which we compute the curvature and torsion while the second one is a helix for which we find the eigenvalue corresponding to the mean curvature vector field.

\section{Normal almost contact geometry and Frenet curves}

Let $M$ be a $r=2 n+1$-dimensional paracompact manifold and $T M$ its tangent bundle. $\mathcal{F}(M)$ represents the algebra of the differentiable functions on $M$ and $\Gamma(E)$ the $\mathcal{F}(M)$-module of the sections of a vector bundle $E$ over $M$. Throughout this paper, all manifolds and maps are differentiable of class $C^{\infty}$. 
Next, suppose that $M$ is endowed with an almost contact metric structure $(\varphi, \xi, \eta, g)$; the following equalities are satisfied:
a) $\varphi^{2}=-I+\eta \oplus \xi$,
b) $\eta(\xi)=1$,
c) $\eta \circ \varphi=0$,
d) $\varphi(\xi)=0$,
e) $\eta(X)=g(X, \xi), f) g(\varphi X, Y)+g(X, \varphi Y)=0, \forall X, Y \in \Gamma(T M)$,

where $I$ is the identity of the tangent bundle $T M$ of $M, \varphi$ is a tensor field of $(1,1)$-type, $\eta$ is a 1 -form, $\xi$ is a vector field on $M$ and $g$ is a Riemannian metric on $M . \xi$ is called the Reeb vector field or the structural vector field. Let $\nabla$ be the Levi-Civita connection of $g$.

The Nijenhuis tensor field with respect to the tensor field $\varphi$, denoted by $N_{\varphi}$, is given by

$$
N_{\varphi}(X, Y)=[\varphi(X), \varphi(Y)]+\varphi^{2}([X, Y])-\varphi([\varphi(X), Y])-\varphi([X, \varphi(Y)]) .
$$

Definition 2.1. The almost contact metric manifold $M(\varphi, \xi, \eta, g)$ is said to be normal if the almost complex structure $J$ on the manifold $M \times \mathbb{R}$, given by

$$
J\left(X, \lambda \frac{d}{d t}\right)=\left(\varphi(X)-\lambda \xi, \eta(X) \frac{d}{d t}\right), X \in \Gamma(T M), \quad t \in \mathbb{R},
$$

is integrable, where $\lambda$ is a real valued function on $M \times \mathbb{R}$.

The condition (2.1) is equivalent to

$$
N_{\varphi}+2 d \eta \otimes \xi=0 .
$$

In the following, we restrict to the dimension $r=3$ for which we have, $[17$, p. 930]:

$$
\left\{\begin{array}{l}
\nabla_{X} \xi=\alpha(X-\eta(X) \xi)-\beta \varphi(X) . \\
\left(\nabla_{X} \varphi\right) Y=\alpha(g(\varphi X, Y) \xi-\eta(Y) \varphi X)+\beta(g(X, Y) \xi-\eta(Y) X) .
\end{array}\right.
$$

where $\alpha=\frac{1}{2} \operatorname{div} \xi$ and $\beta=\frac{1}{2} \operatorname{trace}(\varphi \nabla \xi)$. For the proof of these expressions we refer to [16] where these results appear firstly. An important consequence of $(2.2 a)$ is that $\xi$ is a geodesic vector field: $\nabla_{\xi} \xi=0$; also, with respect to (2.2a) the first term may be called the Kenmotsu part, while the second term may be called the Sasakian part.

Next we recall the notion of a Frenet curve following [4, p. 164]: let $m$ be an integer with $1 \leq m \leq 2 n+1$. The curve $\gamma: I \subseteq \mathbb{R} \rightarrow M$ parametrized by the arc length $s$ is called $m$-Frenet curve on $M$ if there exist $m$ orthonormal vector fields $E_{1}=\gamma^{\prime}, E_{2}, \ldots, E_{m}$ along $\gamma$ so that there exist positive smooth functions $k_{1}, \ldots, k_{m-1}$ of $s$ so that:

$$
\nabla_{\gamma^{\prime}} E_{1}=k_{1} E_{2}, \nabla_{\gamma^{\prime}} E_{2}=-k_{1} E_{1}+k_{2} E_{3}, \ldots, \nabla_{\gamma^{\prime}} E_{m}=k_{m-1} E_{m-1} .
$$

The function $k_{j}$ is called the $j$-th curvature of $\gamma$ while $\gamma$ is:

a) a geodesic if $m=1$; then we get the well-known equation $\nabla_{\gamma^{\prime}} \gamma^{\prime}=0$;

b) a circle if $m=2$ and $k_{1}$ is a constant: then we have $\nabla_{\gamma^{\prime}} E_{1}=k_{1} E_{2}$, $\nabla_{\gamma^{\prime}} E_{2}=-k_{1} E_{1}$

c) a helix of order $m$ if $k_{1}, \ldots, k_{m-1}$ are constants.

The Frenet curve $\gamma$ is called non-geodesic if $k_{1}>0$ everywhere on $I$ and in dimension 3 it is called a generalized helix if $\frac{k_{2}}{k_{1}}=$ const. 
C. Călin and M. Crasmareanu

\section{Slant curves in 3-dimensional normal almost contact geometry}

Return now to $r=3$ and let $\gamma$ be a 3-Frenet curve for which we denotes the Frenet frame as usual $\left(T=\gamma^{\prime}, N, B\right)$ and the Frenet equations:

$$
\nabla_{T} T=k N, \nabla_{T} N=-k T+\tau B, \nabla_{T} B=-\tau N,
$$

where $k$ denotes the curvature and $\tau$ the torsion.

\section{Definition 3.1.}

i) The structural angle of $\gamma$ is the function $\theta: I \rightarrow[0,2 \pi)$ given by

$$
\cos \theta(s)=g(T(s), \xi)=\eta(T(s)) .
$$

The curve $\gamma$ is a slant curve, or more precisely $\theta$-slant curve, if $\theta$ is a constant function, $\left[9\right.$, p. 361]. In the particular case of $\theta=\frac{\pi}{2}$ or $\theta=\frac{3 \pi}{2}$ the curve $\gamma$ is called Legendre curve, [1].

ii) For a non-geodesic $\theta$-slant curve $\gamma$ its Lancret invariant is defined as:

$$
\operatorname{Lancret}(\gamma)=\frac{\cos \theta}{|\sin \theta|} \text {. }
$$

A characterization of these curves and an "a-priori" estimate follows:

Proposition 3.2. The Frenet curve $\gamma$ is a $\theta$-slant curve if and only if, along $\gamma$ the following relation holds:

$$
\eta(N)=-\frac{\alpha}{k} \sin ^{2} \theta
$$

If $\alpha>0$ then a necessary condition for $\gamma$ to be $\theta$-slant is:

$$
|\sin \theta| \leq \min \left\{\sqrt{\frac{k}{\alpha}}, \frac{k}{\alpha}, 1\right\} .
$$

Proof. Let us take the covariant derivative in the relation (3.1) along $\gamma$ :

$$
\begin{aligned}
0 & =-\theta^{\prime} \sin \theta=g(k N, \xi)+g(T, \alpha(T-\eta(T) \xi)+\beta \varphi(T)) \\
& =k \eta(N)+\alpha \sin ^{2} \theta,
\end{aligned}
$$

which yields (3.2) since $\varphi$ is a $g$-skew-symmetric operator. The expression of $\xi$ in the Frenet frame is

$$
\xi=(\cos \theta) T+\left(-\frac{\alpha}{k} \sin ^{2} \theta\right) N+\eta(B) B
$$

and since $\xi$ is an unitary vector field we get that

$$
1=\cos ^{2} \theta+\frac{\alpha^{2}}{k^{2}} \sin ^{4} \theta+\eta(B)^{2}
$$

and then $\eta^{2}(B) \geq 0$ implies $\left|\frac{\alpha}{k} \sin \theta\right| \leq 1$. This last equation together with $\left|\frac{\alpha}{k} \sin ^{2} \theta\right| \leq 1$ given by (3.2) means exactly (3.3) since $\alpha$ and $k$ are strictly positive. 
Remark 3.3.

i) It is important to point out that the characterization (3.2) (as well as (3.3)) does not depend on $\beta$ i.e. does not depend on the Sasakian part.

ii) In $[13$, p. 155$]$ it is introduced the following notion: a non-geodesic curve is called a slant helix if the principal normal lines of $\gamma$ make a constant angle with a fixed direction. Therefore, a slant curve with $\frac{k}{\alpha}=$ constant is a slant helix with $\xi$ as fixed direction.

In the following we suppose that $\gamma$ is non-geodesic i.e. $k>0$ and then $\gamma$ can not be an integral curve of $\xi$ which means $\theta \neq 0, \pi$. From $(2.2 a)$ we have: $\nabla_{\gamma^{\prime}} \xi=\alpha\left(\gamma^{\prime}-\cos \theta \xi\right)-\beta \varphi\left(\gamma^{\prime}\right)$ and then we consider an orthonormal frame field in $T M$ along $\gamma$ :

$$
F_{1}=T=\gamma^{\prime}, F_{2}=\frac{\varphi\left(\gamma^{\prime}\right)}{|\sin \theta|}, F_{3}=\frac{\xi-\cos \theta \gamma^{\prime}}{|\sin \theta|} .
$$

The decomposition of $\xi$ with respect to this frame is:

$$
\xi=\cos \theta F_{1}+|\sin \theta| F_{3} .
$$

The equations of motion for this orthonormal field of frames are:

Proposition 3.4. If $\gamma$ is a $\theta$-slant curve in the normal almost contact metric manifold $M_{3}$ then:

$$
\left\{\begin{array}{l}
\nabla_{\gamma^{\prime}} F_{1}=\delta|\sin \theta| F_{2}-\alpha|\sin \theta| F_{3}, \\
\nabla_{\gamma^{\prime}} F_{2}=-\delta|\sin \theta| F_{1}+(\beta+\delta \cos \theta) F_{3}, \\
\nabla_{\gamma^{\prime}} F_{3}=\alpha|\sin \theta| F_{1}-(\beta+\delta \cos \theta) F_{2},
\end{array}\right.
$$

where

$$
\delta=\frac{1}{\sin ^{2} \theta} g\left(\nabla_{\gamma^{\prime}} \gamma^{\prime}, \varphi\left(\gamma^{\prime}\right)\right)
$$

Also:

$$
\nabla_{\gamma^{\prime}} \xi=\alpha \sin ^{2} \theta F_{1}-\beta|\sin \theta| F_{2}-\alpha \cos \theta|\sin \theta| F_{3} .
$$

We are ready for the first main result of this paper:

Proposition 3.5. The curvature and torsion of a $\theta$-slant curve in $M_{3}$ are:

$$
\left\{\begin{array}{l}
k=|\sin \theta| \sqrt{\alpha^{2}+\delta^{2}}, \\
\tau=\left|\beta+\delta \cos \theta+\frac{\alpha \delta^{\prime}-\alpha^{\prime} \delta}{\alpha^{2}+\delta^{2}}\right| .
\end{array}\right.
$$

The associated Lancret invariant is then, for $\delta \neq 0$ :

$$
\operatorname{Lancret}_{ \pm}(\gamma)=\frac{\left(\alpha^{\prime} \delta-\alpha \delta^{\prime}\right)\left(\alpha^{2}+\delta^{2}\right)^{-\frac{1}{2}}+( \pm \tau-\beta)\left(\alpha^{2}+\delta^{2}\right)^{\frac{1}{2}}}{\delta k} .
$$

Proof. The relation (3.5a) follows from (3.4a) taking into account the definition of the curvature. It follows:

$$
N=\frac{1}{k} \nabla_{\gamma^{\prime}} \gamma^{\prime}=\frac{1}{\sqrt{\alpha^{2}+\delta^{2}}}\left(\delta F_{2}-\alpha F_{3}\right) .
$$




\section{Călin and M. Crasmareanu}

Differentiating the above relation along $\gamma$ we deduce that:

$$
\nabla_{\gamma^{\prime}} N=-k T+\left(\beta+\delta \cos \theta+\frac{\alpha \delta^{\prime}-\alpha^{\prime} \delta}{\alpha^{2}+\delta^{2}}\right) \frac{1}{\sqrt{\alpha^{2}+\delta^{2}}}\left(\alpha F_{2}+\delta F_{3}\right) .
$$

So, denoting

we get

$$
\mu=\beta+\delta \cos \theta+\frac{\alpha \delta^{\prime}-\alpha^{\prime} \delta}{\alpha^{2}+\delta^{2}}
$$

$$
B=\frac{\mu}{|\mu| \sqrt{\alpha^{2}+\delta^{2}}}\left(\alpha F_{2}+\delta F_{3}\right)
$$

and $\tau=|\mu|$.

Let us point out that since $k>0$ the fraction from the expression $(3.5 b)$ is well-defined. Regarding (3.6) the sign corresponds to the sign of $\sin \theta$ according to:

$$
\operatorname{Lancret}_{ \pm}(\gamma)=\frac{\cos \theta}{|\sin \theta|}=\frac{\cos \theta}{ \pm \sin \theta} .
$$

The computation of right hand side of (3.6) is by expressing $\cos \theta$ and $\sin \theta$ from (3.5).

With respect to the Frenet frame we have the decompositions:

$$
\begin{gathered}
F_{2}=\frac{\delta}{\sqrt{\alpha^{2}+\delta^{2}}} N+\frac{\alpha|\mu|}{\mu \sqrt{\alpha^{2}+\delta^{2}}} B, F_{3}=\frac{-\alpha}{\sqrt{\alpha^{2}+\delta^{2}}} N+\frac{\delta|\mu|}{\mu \sqrt{\alpha^{2}+\delta^{2}}} B \\
\xi=\cos \theta T-\frac{\alpha|\sin \theta|}{\sqrt{\alpha^{2}+\delta^{2}}} N+\frac{\delta|\mu||\sin \theta|}{\mu \sqrt{\alpha^{2}+\delta^{2}}} B \\
\nabla_{\gamma^{\prime}} \xi=\alpha \sin ^{2} \theta T+\frac{|\sin \theta|}{\sqrt{\alpha^{2}+\delta^{2}}}\left[\left(\alpha^{2} \cos \theta-\beta \delta\right) N-\frac{(\beta+\delta \cos \theta) \alpha|\mu|}{\mu} B\right] .
\end{gathered}
$$

Then the norm of $\nabla_{\gamma^{\prime}} \xi$ is independent of $\gamma:\left\|\nabla_{\gamma^{\prime}} \xi\right\|=|\sin \theta| \sqrt{\alpha^{2}+\beta^{2}}$.

Remark 3.6. Some particular cases are as follows:

1. ( $\beta$-Sasakian). For $\alpha=0$ and $\beta \neq 0$ we get the $\beta$-Sasakian case and in this way we generalize the results obtained for $\beta=1$ in [9]. For example, Proposition 3.1 of $[9$, p. 361] is true for every $\beta$. Also, the Lancret invariant (1.1) admits the following generalization: a non-geodesic slant curve in a $\beta$-Sasakian 3 -manifold has the constant ratio $\frac{\tau \pm \beta}{k}$ which can be considered the Lancret invariant in $\beta$-Sasakian geometry.

2. $(\alpha$-Kenmotsu $)$. For $\beta=0$ and $\alpha \neq 0$ we recover the results obtained in [8] where $\alpha$ is denoted $f$.

3. If $\alpha=\beta=0$ then we have the cosymplectic case for which (3.5) becomes: $k=|\delta \sin \theta|, \tau=|\delta \cos \theta|$ and $\operatorname{Lancret}_{ \pm}(\gamma)= \pm \frac{\tau}{k}$ which can be put in correspondence with (1.1) of Introduction. Therefore, a slant curve in a cosymplectic manifold is a generalized helix.

4. For $\theta=\frac{\pi}{2}$ we get the case of Legendre curve studied by Joanna Welyczko in $[17]$. 
Now, denote by $h$ the second fundamental form of $\gamma$ and by $H$ its mean curvature field. We know that

$$
H=\operatorname{trace}(h)=h(T, T)=\nabla_{T} T .
$$

Then $\gamma$ is called a curve with proper mean curvature vector field if there exists $\lambda \in C^{\infty}(\gamma)$ so that

$$
\Delta H=\lambda H
$$

In particular, if $\lambda=0$ then $\gamma$ is known as a curve with harmonic mean curvature vector field. Here the Laplace operator $\Delta$ acts on the vector valued function $H$ and it is given by

$$
\Delta H=-\nabla_{T} \nabla_{T} \nabla_{T} T
$$

Making use of Frenet equations, we can rewrite (3.7) as

$$
-3 k^{\prime} k T+\left(k^{\prime \prime}-k^{3}-k \tau^{2}\right) N+\left(2 k^{\prime} \tau+k \tau^{\prime}\right) B=-\lambda k N .
$$

It follows that both $k$ and $\tau$ are constants, and the function $\lambda$ becomes a constant too, namely

$$
\lambda=\kappa^{2}+\tau^{2}
$$

(see also Theorem 1.1 in [12]).

For our setting we state the following:

Proposition 3.7. A non-geodesic $\theta$-slant curve $\gamma$ in $M_{3}$ has a proper mean curvature vector field if and only if $\gamma$ is a helix and then:

$$
\lambda=\alpha^{2} \sin ^{2} \theta+\beta^{2}+\delta^{2}+\left(\alpha^{\prime}\right)^{2}+2 \beta \delta \cos \theta-2 \beta \alpha^{\prime}-2 \delta \alpha^{\prime} \cos \theta .
$$

In particular, a helix Legendre curve has

$$
\lambda=\alpha^{2}+\delta^{2}+\left(\alpha^{\prime}-\beta\right)^{2}>0 .
$$

Proof. We compute $\lambda$ of (3.8) by using (3.5) and obtain:

$$
\begin{aligned}
\lambda= & \alpha^{2} \sin ^{2} \theta+\beta^{2}+\delta^{2}+\left(\frac{\alpha \delta^{\prime}-\alpha^{\prime} \delta}{\alpha^{2}+\delta^{2}}\right)^{2}+2 \beta \delta \cos \theta \\
& +2 \frac{\beta\left(\alpha \delta^{\prime}-\alpha^{\prime} \delta\right)}{\alpha^{2}+\delta^{2}}+2 \frac{\delta\left(\alpha \delta^{\prime}-\alpha^{\prime} \delta\right)}{\alpha^{2}+\delta^{2}} \cos \theta
\end{aligned}
$$

But the constancy of $k$ gives, for $\delta \neq 0$,

$$
\delta^{\prime}=-\frac{\alpha \alpha^{\prime}}{\delta},
$$

which replaced in (3.10) yields (3.9). If $\delta=0$ then $k=\alpha|\sin \theta|$ and $\tau=|\beta|$ and from the helix property it follows the same formula (3.9) reduced to: $\lambda=\alpha^{2} \sin ^{2} \theta+\beta^{2}$. 


\section{Călin and M. Crasmareanu}

\section{Examples}

Let $N$ be an open connected subset of $\mathbb{R}^{2},(a, b)$ an open interval in $\mathbb{R}$ and let us consider the manifold $M=N \times(a, b)$. Let $(x, y)$ be the coordinates on $N$ induced from the cartesian coordinates on $\mathbb{R}^{2}$ and let $z$ be the coordinate on $(a, b)$ induced from the cartesian coordinate on $\mathbb{R}$. Thus $(x, y, z)$ are the coordinates on $M$. Now we choose the functions

$$
\omega_{1}, \omega_{2}: N \rightarrow \mathbb{R}, \quad \sigma, f: M \rightarrow \mathbb{R}_{+}^{*},
$$

and following the idea from [17] we define a normal almost contact metric structure $(\varphi, \xi, \eta, g)$ on $M$ as follows:

$$
\begin{gathered}
\varphi\left(\frac{\partial}{\partial x}\right)=\frac{\partial}{\partial y}-\omega_{2} \frac{\partial}{\partial z}, \varphi\left(\frac{\partial}{\partial y}\right)=-\frac{\partial}{\partial x}+\omega_{1} \frac{\partial}{\partial z}, \varphi\left(\frac{\partial}{\partial z}\right)=0, \eta=d z+\omega_{1} d x+\omega_{2} d y, \\
g=\left[g_{i j}\right]=\left[\begin{array}{ccc}
\omega_{1}^{2}+\sigma e^{2 f} & \omega_{1} \omega_{2} & \omega_{1} \\
\omega_{1} \omega_{2} & \omega_{2}^{2}+\sigma e^{2 f} & \omega_{2} \\
\omega_{1} & \omega_{2} & 1
\end{array}\right] .
\end{gathered}
$$

Next we choose a $g$-orthonormal field of frames on $M$ as follows:

$$
H_{1}=\frac{e^{-f}}{\sqrt{\sigma}}\left[\frac{\partial}{\partial x}-\omega_{1} \frac{\partial}{\partial z}\right], H_{2}=\frac{e^{-f}}{\sqrt{\sigma}}\left[\frac{\partial}{\partial y}-\omega_{2} \frac{\partial}{\partial z}\right], \quad H_{3}=\xi=\frac{\partial}{\partial z} .
$$

We get

$$
\left\{\begin{array}{l}
{\left[H_{1}, H_{2}\right]=\frac{\sqrt{\sigma}}{e^{-f}}\left[-H_{2}\left(\frac{e^{-f}}{\sqrt{\sigma}}\right) H_{1}+H_{1}\left(\frac{e^{-f}}{\sqrt{\sigma}}\right) H_{2}\right]+\frac{e^{-2 f}}{\sigma}\left(\frac{\partial \omega_{1}}{\partial y}-\frac{\partial \omega_{2}}{\partial x}\right) H_{3},} \\
{\left[H_{1}, H_{3}\right]=\frac{\sigma_{z}}{2 \sigma} H_{1}, \quad\left[H_{2}, H_{3}\right]=\frac{\sigma_{z}}{2 \sigma} H_{2} .}
\end{array}\right.
$$

Here, the subscript denotes the variable with respect to which we derive. It follows that

$$
\alpha=\frac{1}{2 \sigma} \frac{\partial \sigma}{\partial z}+\frac{\partial f}{\partial z}, \quad \beta=\frac{e^{-2 f}}{2 \sigma}\left(\frac{\partial \omega_{1}}{\partial y}-\frac{\partial \omega_{2}}{\partial x}\right)
$$

and then for $\sigma=\sigma(x, y)$ we get the $\beta$-Sasakian case while $\eta$ is closed if and only if $\beta=0$ i.e. we have the $\alpha$-Kenmotsu case. Let us point out that in [17] the function $f$ is considered only as $f=f(x, y)$ and then in the expression of $\alpha$ the term in $\frac{\partial f}{\partial z}$ disappear.

If we denote $\gamma(s)=\left(\gamma_{1}(s), \gamma_{2}(s), \gamma_{3}(s)\right)$ then $\gamma$ is a $\theta$-slant curve if and only if

$$
\left\{\begin{array}{l}
\omega_{1} \gamma_{1}^{\prime}+\omega_{2} \gamma_{2}^{\prime}+\gamma_{3}^{\prime}=\cos \theta \\
\left(\omega_{1}^{2}+\sigma e^{2 f}\right)\left(\gamma_{1}^{\prime}\right)^{2}+\left(\omega_{2}^{2}+\sigma e^{2 f}\right)\left(\gamma_{2}^{\prime}\right)^{2}+\left(\gamma_{3}^{\prime}\right)^{2} \\
\quad+2 \omega_{1} \omega_{2} \gamma_{1}^{\prime} \gamma_{2}^{\prime}+2 \omega_{1} \gamma_{1}^{\prime} \gamma_{3}^{\prime}+2 \omega_{2} \gamma_{2}^{\prime} \gamma_{3}^{\prime}=1 .
\end{array}\right.
$$

But (4.2b) becomes

$$
\left(\omega_{1} \gamma_{1}^{\prime}+\omega_{2} \gamma_{2}^{\prime}+\gamma_{3}^{\prime}\right)^{2}+\sigma e^{2 f}\left[\left(\gamma_{1}^{\prime}\right)^{2}+\left(\gamma_{2}^{\prime}\right)^{2}\right]=1
$$


and then $\gamma$ is a $\theta$-slant curve if and only if

$$
\left\{\begin{array}{l}
\omega_{1} \gamma_{1}^{\prime}+\omega_{2} \gamma_{2}^{\prime}+\gamma_{3}^{\prime}=\cos \theta \\
\left(\gamma_{1}^{\prime}\right)^{2}+\left(\gamma_{2}^{\prime}\right)^{2}=\frac{\sin ^{2} \theta}{\sigma} e^{-2 f} .
\end{array}\right.
$$

Next it is easy to see that

$$
\gamma^{\prime}=\sqrt{\sigma} e^{f}\left(\gamma_{1}^{\prime} H_{1}+\gamma_{2}^{\prime} H_{2}\right)+\cos \theta H_{3}
$$

and

$$
\varphi\left(\gamma^{\prime}\right)=\sqrt{\sigma} e^{f}\left(-\gamma_{2}^{\prime} H_{1}+\gamma_{1}^{\prime} H_{2}\right) .
$$

Now, we can derive the general expression of slant curves for this general class of manifolds:

Proposition 4.1. Let $\gamma$ be a non-geodesic $\theta$-slant curve in $(M, \varphi, \xi, \eta, g)$. Then $\gamma$ is given by

$\gamma(s)=|\sin \theta|\left(\int_{s_{0}}^{s} \frac{e^{-} f}{\sqrt{\sigma}} \zeta(t) d t, s \frac{\cos \theta}{|\sin \theta|}-\int_{s_{0}}^{s} \frac{e^{-} f}{\sqrt{\sigma}}\left(\omega_{1} \cos u(t)+\omega_{2} \sin u(t)\right) d t\right)$,

where $\zeta(s)=(\cos u(s), \sin u(s))$ is an arbitrary smooth parametrization of the circle $\mathbb{S}^{1}$.

Proof. From (4.3b) follows the existence of a function $u=u(s)$ so that

$$
\left\{\begin{array}{l}
\gamma_{1}^{\prime}=\frac{|\sin \theta|}{\sqrt{\sigma}} e^{-f} \cos u(s), \\
\gamma_{2}^{\prime}=\frac{|\sin \theta|}{\sqrt{\sigma}} e^{-f} \sin u(s)
\end{array}\right.
$$

which, replaced in $(4.3 a)$ yields:

$$
\gamma_{3}^{\prime}=\cos \theta-\frac{|\sin \theta|}{\sqrt{\sigma}} e^{-f}\left(\omega_{1} \cos u(s)+\omega_{2} \sin u(s)\right) .
$$

So, the conclusion follows directly.

Now, we make the choice of Example 1 of $\left[17\right.$, p. 936]: $N=\mathbb{R}^{2},(a, b)=$ $\mathbb{R}_{+}, \omega_{1}=f=0, \omega_{2}=2 x$ and $\sigma=2 z$ which gives that $M=\mathbb{R}^{2} \times \mathbb{R}_{+}$is normal but not quasi-Sasakian; more precisely is trans-Sasakian ([7]). Then, from (4.1) it follows:

$$
\alpha=\frac{1}{2 z}=-\beta .
$$

Also, we choose $\gamma_{1}=0$ which means that the function $u$ is a constant, namely $u=\frac{\pi}{2}$. From $(4.3 a)$ since $\left.\omega_{2}\right|_{\gamma}=0$ it follows $\gamma_{3}=\cos \theta s$ and from (4.4b) we get a non-Legendre curve on $I=(0,+\infty)$ :

$$
\gamma_{2}^{\prime}=\frac{|\sin \theta|}{\sqrt{2 \cos \theta}} \cdot \frac{1}{\sqrt{s}},
$$

with solution

$$
\gamma_{2}=|\sin \theta| \sqrt{\frac{2 s}{\cos \theta}}
$$




\section{Călin and M. Crasmareanu}

and then the $\theta$-slant curve is

$$
\gamma_{ \pm}(s)=\left(0, \pm \sin \theta \sqrt{\frac{2 s}{\cos \theta}}, \cos \theta s\right) .
$$

A long but straightforward computation using the frame $\left\{H_{1}, H_{2}, H_{3}\right\}$ and the Koszul formula for $\nabla$ and this frame gives

$$
\delta(s)=\frac{1}{s}
$$

and then

$$
\left\{\begin{array}{l}
k=\frac{|\sin \theta|}{2 s \cos \theta} \sqrt{1+4 \cos ^{2} \theta}, \\
\tau=\frac{|\cos 2 \theta|}{2 s \cos \theta} .
\end{array}\right.
$$

The curve is a generalized helix with

$$
\frac{\tau}{k}=\frac{|\cos 2 \theta|}{|\sin \theta| \sqrt{1+4 \cos ^{2} \theta}} .
$$

In the same manifold $(M, \varphi, \xi, \eta, g)$ an example of a helix Legendre curve is given in $[17$, p. 936] again on $I=(0,+\infty)$ :

$$
\gamma_{3}(s)=\left(0, \frac{s}{2}, 2\right) \text {, }
$$

with

$$
k=\tau=\frac{1}{4} .
$$

From (3.5a) and $\alpha=-\beta=\frac{1}{4}$ it follows $\delta=0$ and then we apply the Proposition 2.7: $\gamma_{3}$ has a proper mean curvature vector field with:

$$
\lambda=\frac{1}{8} .
$$

\section{Acknowledgment}

The authors cordially thank the referee(s) for several useful remarks and corrections about the initial Submission. The comments by Professor Aurel Bejancu are deeply appreciated.

\section{References}

[1] Ch. Baikoussis and D. E. Blair, On Legendre curves in contact 3-manifolds, Geom. Dedicata 49 (no. 2) (1994), 135-142.

[2] M. Barros, General helices and a theorem of Lancret, Proc. Amer. Math. Soc. 125 (no. 5) (1997), 1503-1509.

[3] M. Belkhelfa, I.-E. Hirică, R. Rosca and L. Verstraelen, On Legendre curves in Riemannian and Lorentzian Sasaki spaces, Soochow J. Math. 28 (no. 1) (2002), 81-91.

[4] D. E. Blair, Riemannian geometry of contact and symplectic manifolds, second edition, Progress in Mathematics, 203. Birkhäuser Boston, Inc., Boston, MA, 2010 . 
[5] D. E. Blair, F. Dillen, L. Verstraelen and L. Vrancken, Deformations of Legendre curves, Note Mat. 15 (no. 1) (1995), 99-110.

[6] C. Camci, Y. Yayli and H. H. Hacisalihoglu, On the characterization of spherical curves in 3-dimensional Sasakian spaces, J. Math. Anal. Appl. 342 (no. 2) (2008), 1151-1159.

[7] C. Călin, Foliations and complemented framed structures on an almost contact metric manifold, Mediterr. J. Math. 8 (no. 2) (2011), 191-206. MR2802323

[8] C. Călin, M. Crasmareanu and M.-I. Munteanu, Slant curves in 3-dimensional f-Kenmotsu manifolds, J. Math. Anal. Appl. 394 (no. 1) (2012), 400-407.

[9] J. T. Cho, J.-I. Inoguchi and J.-E. Lee, On slant curves in Sasakian 3manifolds, Bull. Austral. Math. Soc. 74 (no. 3) (2006), 359-367.

[10] J. T. Cho and J.-E. Lee, Slant curves in contact pseudo-Hermitian 3-manifolds, Bull. Aust. Math. Soc. 78 (no. 3) (2008), 383-396.

[11] D. Fetcu, Biharmonic Legendre curves in Sasakian space forms, J. Korean Math. Soc. 45 (no. 2) (2008), 393-404.

[12] J.-I. Inoguchi, Submanifolds with harmonic mean curvature vector field in contact 3-manifolds, Colloq. Math. 100 (no. 2) (2004), 163-179.

[13] S. Izumiya and N. Takeuchi, New special curves and developable surfaces, Turkish J. Math. 28 (no. 2) (2004), 153-163.

[14] J.-E. Lee, On Legendre curves in contact pseudo-Hermitian 3-manifolds, Bull. Aust. Math. Soc. 81 (no. 1) (2010), 156-164.

[15] K. Smoczyk, Closed Legendre geodesics in Sasaki manifolds, New York J. Math. 9 (2003), 23-47.

[16] Z. Olszak, Normal almost contact metric manifolds of dimension three, Ann. Pol. Math., 47 (no. 1) (1986), 41-50.

[17] J. Welyczko, On Legendre curves in 3-dimensional normal almost contact metric manifolds, Soochow J. Math. 33 (no. 4) (2007), 929-937.

[18] J. Welyczko, On Legendre curves in 3-dimensional normal almost paracontact metric manifolds, Results Math. 54 (no. 3-4) (2009), 377-387.

Constantin Călin

Technical University "Gh. Asachi"

Department of Mathematics

700049, Iaşi

Romania

e-mail: c0nstc@yahoo.com

Mircea Crasmareanu

University "Al. I. Cuza"

Faculty of Mathematics

700506, Iaşi

Romania

e-mail: mcrasm@uaic.ro

Received: January 27, 2012.

Revised: April 25, 2012.

Accepted: June 4, 2012. 